\title{
Parasite mediated homogenizing selection at the MHC in guppies
}

\author{
Bonnie A. Fraser · Bryan D. Neff
}

Received: 8 April 2009/Accepted: 14 August 2009/Published online: 29 August 2009

(C) Springer Science+Business Media B.V. 2009

\begin{abstract}
Understanding the selective forces influencing genetic diversity is a fundamental goal of evolutionary ecology. The genes of the major histocompatibility complex (MHC) play a key role in the adaptive immune response of vertebrates and thus provide an excellent opportunity to examine the agents of selection on a functionally important gene. Here we examine the genetic architecture of the MHC class IIB genes in 10 wild populations of guppies (Poecilia reticulata) in Northern Trinidad. We have previously shown that these populations are significantly less diverged at the class IIB locus than expected based on neutral (microsatellite) loci. We now survey infection by Gyrodactylus turnbulli and G. bullatarudis, common parasitic worms that infect guppies, as a potential agent of homogenizing selection. We used a genetic algorithm to partition both additive and non-additive genetic effects of the five most common MHC allele types as well as a rare allele category. Although we found no evidence for non-additive effects, across the populations we found that one allele type (the a-type) had a significant negative additive effect on parasite load. Thus, individuals who had more copies of the a-type allele were infected with fewer gyrodactylus than individuals with fewer copies of the allele. These results not only link parasite infection with MHC genotype, they provide a mechanism of homogenizing selection across these otherwise disparate populations.
\end{abstract}

Keywords Major histocompatibility complex . Poecilia reticulata Parasite selection · Gyrodactylus

B. A. Fraser $(\bowtie) \cdot$ B. D. Neff

Department of Biology, University of Western Ontario, 1151 Richmond Street, London, Ontario N6A 5B7, Canada e-mail: fraser.bonnie8@gmail.com

\section{Introduction}

The ecological processes that shape genetic divergence among and within species are a key area of research in biology. The Trinidadian guppy (Poecilia reticulata) remains an extensively studied system because of the rapid divergent evolution among populations. For example, guppy populations differ predictably in morphological and behavioural traits in response to selection from different predation regimes (Houde 1997). Additionally, there is a high degree of genetic divergence at neutral loci among populations from different drainages (Carvahlo et al. 1991) and even populations within rivers (Suk and Neff 2009). Paradoxically, despite the adaptive and neutral divergence among guppy populations, strong reproductive isolation or other signs of incipient speciation among guppy populations appear to be absent (reviewed in Magurran 1998).

Recently, it has been shown that the MHC class IIB, an important gene in the adaptive immune system, is not as diverged across guppy populations in Northern Trinidad as microsatellite loci (van Oosterhout et al. 2006; Fraser et al. 2009). Here we hypothesize that pathogens are acting as a homogenizing selective agent at this important fitness locus and preventing genetic divergence across populations. $G y$ rodactylus turnbulli and G. bullatarudis (hereafter referred to as gyrodactylus) are major pathogens of wild guppies and they are found in many populations across different ecological factors and drainages (Lyles 1990; Martin and Johnsen 2007). Thus, gyrodactylus are an excellent candidate as an agent of homogenizing selection in the guppy. Gyrodactylus are small (ca. $0.5 \mathrm{~mm}$ long) monogenean fluke parasites that attach to the integument of their host and feed on its protective mucus and skin. The exponential population growth of gyrodactylus on their host, due to their short generation time and direct life cycle, can lead to a 
damaging or even lethal infection in guppies (e.g. Scott and Anderson 1984). Indeed, van Oosterhout et al. (2007a), using a mark recapture experiment, have provided direct evidence of gyrodactylus mediated selection.

Both innate and adaptive resistance to gyrodactylus has been shown to be heritable in the guppy (Scott 1985; Cable and van Oosterhout 2007a), but a clear link to MHC variation has yet to be established. In one study, van Oosterhout et al. (2006) surveyed the upper and lower Aripo populations and compared variation of MHC and parasite intensity at the population level. They showed that the observed allele frequencies at the MHC could be maintained by overdominance selection, suggesting that gyrodactylus could be an agent of selection on the MHC; although, recently van Oosterhout (2009) has also argued that the observed diversity could be maintained by selection at closely linked loci. Therefore, it remains to be determined what effect the MHC has on gyrodactylus resistance.

Here, we examine the relationship between MHC variation and parasite resistance in guppies from 10 populations from five rivers and three drainages. We examine MHC allele and genotype effects on parasite infection from gyrodactylus and use these data to examine the potential causative factors of selection on the MHC in the wild.

\section{Materials and methods}

During May of 2006, we collected an average of $41.1 \pm 2.6$ (SD) guppies from 10 populations across Northern Trinidad ( $\mathrm{N}=412$ total). The populations comprised upstream and downstream locales from the Aripo and Guanapo rivers in the Caroni drainage, the Quare and Turure rivers in the Oropouche drainage, and the Yarra river on the northern coast. Fish were individually packaged to avoid cross-contamination and transported back to our lab at the University of the West Indies, St. Augustine where they were housed individually before examination. All fish were analyzed within $24 \mathrm{~h}$ of being collected.

First, fish were lightly anesthetized with MS222 and an external examination was conducted and standard length and sex were recorded. The fish were then euthanized with an overdose of MS222 and a skin smear was taken, two gill arches were excised at random, followed by a whole body dissection as outlined in Roberts and Smail (2001). The number of Gyrodactylus turnbulli and $G$. bullatarudis flukes, without identifying species, on each individual was recorded. The two species have similar pathologies in guppies and infection with either species results in similar parasite load and parasite-related mortality (Cable and van Oostehout 2007b). No flukes were observed on individuals from the upper Guanapo, upper Quare and upper Turure sites and we therefore excluded these populations from analysis of parasite and genotype correlations. No other parasites, besides gyrodactylus, were found in any individual. Other parasites, particularly digeneans, have been reported to infect guppies in these populations but are also known to vary spatially and temporally and may account for the lack of other parasites found in this study (Cable and van Oosterhout 2007a).

Whole body tissue samples were preserved in $95 \%$ ethanol for genetic analysis. From our sample, a subset of fish was chosen randomly for genetic analysis. Briefly, an average of 14 fish from each population (range: 11-17) were genotyped at the MHC class IIB gene using a combination of single strand conformation polymorphism (SSCP), cloning and sequencing (see Fraser et al. 2009 for full details). The MHC class IIB reading frame was determined by aligning our sequences with the reading frame identified for the guppy in van Oosterhout et al. (2006) and three species of African cichlids (Haplochromis lividus, Oreochromis alcalicus, and Aulonocara hansbaenschi) (Figueroa et al. 2000; Ono et al. 1993). The amplified $230 \mathrm{bp}$ were subsequently trimmed to $218 \mathrm{bp}$ and 72 codons that constituted an open reading frame. The MHC class IIB gene is duplicated in the guppy and therefore individuals can have up to four different alleles (McConnell et al. 1998; van Oosterhout et al. 2006).

To determine whether or not individual genes or genotypes were related to gyrodactylus infection (number of flukes) we used an algorithm adapted from Pitcher and Neff (2006). First, alleles that differed by three amino acids or fewer and had unique peptide binding residues were grouped together into an allele "type" and we focused on the five most common allele types; all other types were grouped into a "rare" category. The total number of flukes was transformed using $\log _{10}+1$. The algorithm relates infection to both additive and non-additive genetic effects by using the general linear model:

$\log _{10}($ number of flukes +1$)=\mu+S\left(\mathrm{a}_{\mathrm{i}} \times \mathrm{A}_{\mathrm{i}}\right)+\mathrm{S}\left(\mathrm{n}_{\mathrm{ij}} \times \mathrm{N}_{\mathrm{ij}}\right)$

where $\mu$ is the mean number of flukes infecting individuals, $a_{i}$ is the additive coefficient for allele $i, A_{i}$ is the number of copies of allele $\mathrm{i}, \mathrm{n}_{\mathrm{ij}}$ is the non-additive coefficient associated with alleles $\mathrm{i}$ and $\mathrm{j}$, and $\mathrm{N}_{\mathrm{ij}}$ was coded as either 1 if individuals had at least one copy of both alleles $i$ and $j$ or 0 otherwise. Not all combinations of alleles were observed and therefore some non-additive effects were not assessed. We also did not consider any three-way non-additive effects. First, significance of the general linear model was determined using a bootstrap routine where we randomized parasite load with respect to genotype data and then determined the proportion of runs that the $\mathrm{R}^{2}$ was higher than the $\mathrm{R}^{2}$ of the non-randomized (observed) data. 
Significance of each coefficient was assessed using a bootstrap routine that was repeated for a total of 1,000 samples. Each bootstrap resampled the data with replacement and re-calculated the coefficients; $P$-values were then determined as the proportion of bootstrap runs in which a given coefficient was either above or below zero. We also considered a model that included a population effect to control for any potential spurious association between the frequency of the a-type alleles and intensity of the infection across populations.

\section{Results}

Gyrodactylus infection was found in seven of the 10 populations surveyed (Fig. 1). Across the seven populations that exhibited infection, we found that $25 \%$ of fish were infected with the parasite (range: $10-38 \%, \mathrm{~N}=295$ ).
Infected individuals had an average of $1.96 \pm 0.17$ (SD; range: 1-8) flukes. Standard length of the fish was not related to the number of flukes present (Spearman's $\rho=0.077, \mathrm{~N}=412, P=0.12$ ). Females had more flukes (mean $=0.44 \pm 0.07)$ than males $($ mean $=0.26 \pm 0.07$ ), but this difference was not significant $\left(\mathrm{t}_{410}=1.87\right.$, $P=0.062$ ). Furthermore, there was no difference in the number of males and females analyzed across populations $\left(\chi^{2}=0.24 \mathrm{n}=412 P<0.63\right)$.

We found a total of 39 different amino acid sequences (see Fraser et al. 2009), which were binned into the five most common allele types and one rare type category. Across all populations, the a-type allele was the most common with a mean frequency of 69\% (range: 33-100\%), whereas, the other types of alleles were less common (range: 0-15\%; Fig. 1). The general linear model significantly explained the variation in parasite load (Table 1). The a-type allele had a significant additive effect on the

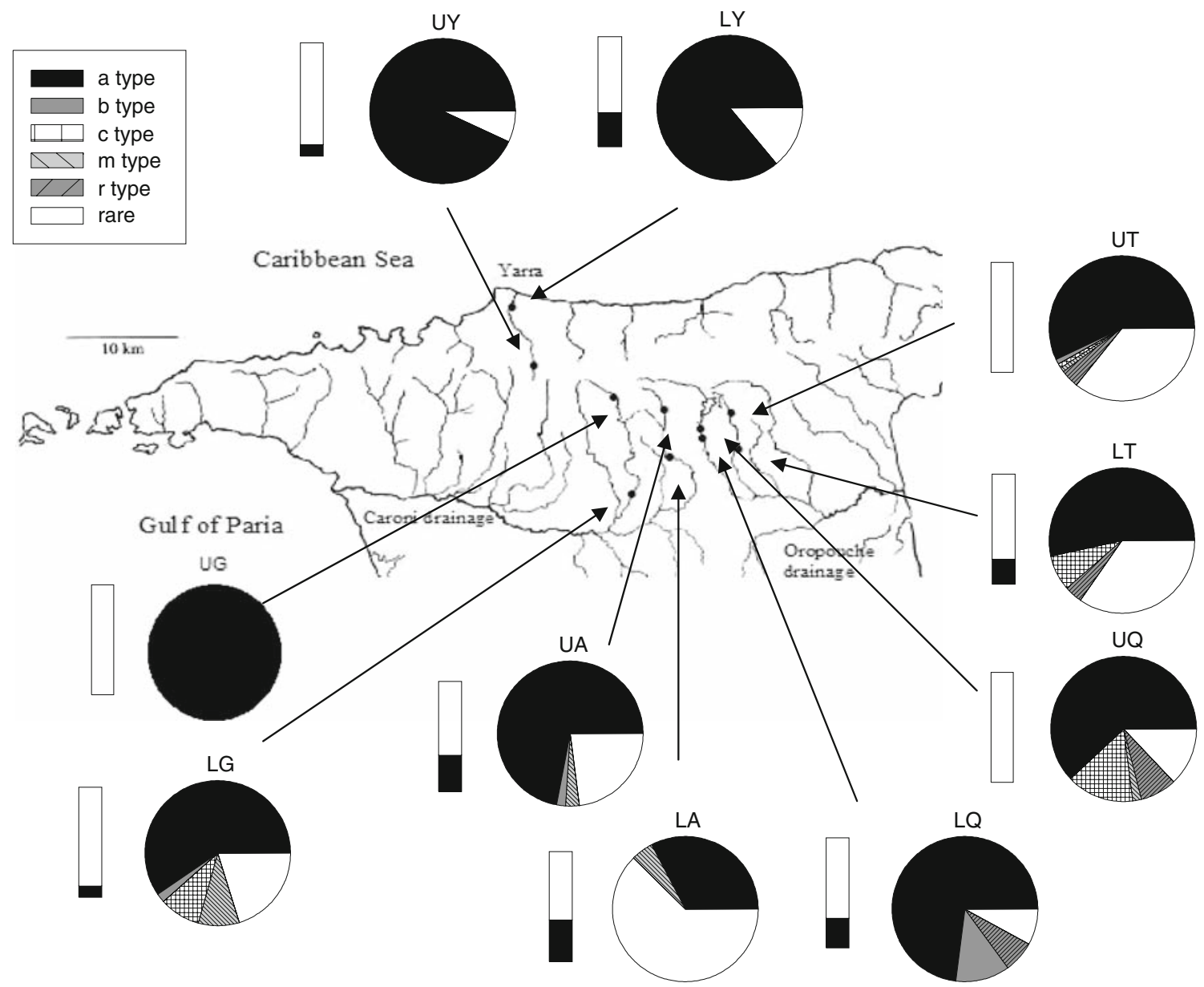

Fig. 1 Location and MHC class IIB allele frequencies of 10 guppy (Poecilia reticulata) sampling sites in Northern Trinidad. Populations comprise Lower Yarra (LY), Upper Yarra (UY), Lower Quare (LQ), Upper Quare (UQ), Lower Turure (LT), Upper Turure (UT), Lower Guanapo (LG), Upper Guanapo (UG), Lower Aripo (LA), and Upper Aripo (UA). Pie charts display the frequencies of the five most common allele types and the remaining rare alleles category for each population. Bar graphs show the proportion of individuals that were parasitized with Gyrodactylus turnbulli and G. bullatarudis flukes, black indicating proportion of individuals parasitized and white indicating the proportion that were not parasitized 
Table 1 Additive and non-additive genetic effects of five MHC class IIB allele types and a rare alleles category on the number of Gyrodactylus turnbulli and $G$. bullatarudis flukes found on guppies (Poecilia reticulata)

\begin{tabular}{llll}
\hline & & Coefficients & $P$ \\
\hline Model & & 0.30 & $\mathbf{0 . 0 0 7}$ \\
Additive effects & a & -0.012 & $\mathbf{0 . 0 1 4}$ \\
& b & -0.037 & 0.12 \\
& c & 0.028 & 0.32 \\
& m & 0.17 & 0.59 \\
Non-additive effects & r & 0.086 & 0.23 \\
& rare & 0.023 & 0.060 \\
& a and b & 0.13 & 0.23 \\
& a and m & 0.066 & 0.47 \\
& a and rare & -0.022 & 0.35 \\
& b and rare & -0.097 & 0.28 \\
& m and rare & -0.12 & 0.42 \\
& r and rare & -0.22 & 0.18 \\
\hline
\end{tabular}

Not all combinations of alleles were observed and therefore some non-additive effects were not assessed. The results include the $\mathrm{R}^{2}$ for the general linear model, the coefficient for each allele or pair of alleles, and the associated p-values (significant values are in bold)

number of flukes (Table 1); individuals with a copy of an a-type allele had fewer flukes $(0.51 \pm 0.15)$ than individuals without an a-type allele $(1.00 \pm 0.33)$. There was no other significant additive or non-additive effect of any other allele type (Table 1). We also confirmed this additive effect of the a-type allele on the incidence of infection (infected $=1$ non-infected $=0$ ) using a logistical ordinal regression. We found that the a-type had a significant negative effect on incidence of infection $\left(\chi^{2}=4.12\right.$, df $=1,98, P=0.042)$. Inclusion of the population effect in the general linear model was not significant $(P=0.59$ for comparison of the $\mathrm{R}^{2}$ to the model without the population term) and did not change the significant negative effect of the a-type allele $(P=0.036$ with the inclusion of the population effect). Across populations, there was also a negative relationship between the frequency of the a-type allele and the frequency of parasite infection, although the relationship was not significant (Spearman's $\rho=-0.30$, $\mathrm{n}=10, P=0.40)$.

\section{Discussion}

Our results show an advantage of the most common MHC class IIB allele type found in the guppy populations from Northern Trinidad. The number of a-type alleles was associated with a decrease in the number of gyrodactylus flukes infecting individual guppies, which indicates an additive effect of the allele type on resistance to gyrodactylus. We found no additive effect of any other type of allele and no non-additive effects of any pair of alleles. Gyrodactylus is a pervasive pathogen and is known to cause serious harm and to be lethal to guppies (e.g. Scott and Anderson 1984; Cable and van Oosterhout 2007a, b; van Oosterhout et al. 2007a, b, 2008). Therefore the a-type allele should confer a strong fitness advantage, which most likely accounts for its high frequency in populations. The hypothesis that the a-type is being maintained by homogenizing selection is further supported by lower pairwise population genetic differentiation at the MHC than at neutral microsatellite loci driven mainly be the high frequency of the a-type alleles (Fraser et al. 2009). Our results do not, however, support those of van Oosterhout et al. (2006), who argued that homogenizing selection in the upper and lower Aripo populations was due to balancing selection resulting from a heterozygote advantage. We found no evidence for non-additive effects on parasite load at least not across the seven populations that had gyrodactylus. Similarly, van Oosterhout (2009), using selection coefficient of gyrodactylid-induced mortality rate estimated from van Oosterhout et al. (2007a), concluded that parasite selection was high but insufficient to explain the level of inferred overdominance selection on the MHC. We conclude that directional selection exerted by gyrodactylus in each population is responsible for the high frequency of the a-type alleles and homogenizing allele frequencies at the MHC in these otherwise genetically disparate guppy populations.

Although the results presented here are correlational, the hypothesis that the a-type allele confers resistance to gyrodactylus is further supported by experimental results from infectivity trials (Fraser and Neff in review). We experimentally infected guppies from a stock population by transferring two flukes and then monitored the spread of the parasite infection on individual fish over the course of 10 days. We found that an a-type allele had a negative additive effect on parasite load. Taken together, these data provide strong evidence that the a-type allele provides resistance to gyrodactylus infection in the guppy.

The established view in fishes has been that response to gyrodactylus infection is provided by the innate immune system. However, evidence is mounting that the adaptive immune system is also important in gyrodactylus resistance. While the innate immune system reacts to infections in a non-specific manner, the adaptive immune system recognizes foreign antigens, through the $\mathrm{MHC}$, and reacts to infections with specificity and memory (Klein 1990). Kania et al. (2007) showed that after infection with $G y$ rodactylus salaris, Baltic Atlantic salmon (Salmo salar) showed increased expression in MHC class I genes (also see Lindenstrøm et al. 2004). In guppies, Scott (1985) found that fish previously infected with gyrodactylus were 
better able to combat infection, even if the previous infection had been 6 weeks prior (also see Cable and van Oosterhout 2007a). Similarly other studies have found resistance conferred by the MHC to other monogenean parasites (Ottová et al. 2007), parasites in the phylum platyhelminths (Wegner et al. 2003), and other parasitic worms known as helminths (Schad et al. 2005). Therefore it is likely that the MHC can recognize antigens from gyrodactylus and mount a reaction using the adaptive immune system. Our results lead us to propose that specific amino acid variants of the MHC contribute to the recognition of gyrodactylus infection and link it to the adaptive immune system and subsequent resistance and clearance of the infection.

A recent model by Eizaguirre et al. (2009) shows how the pleiotropic effect of the MHC in local adaptation and mate choice contexts can accelerate and stabilize speciation among populations. The premise of the authors' model is that local selection on the MHC by the pathogen community differs among populations and mate choice for optimal MHC genotypes then leads to reproductive barriers across the populations. We have instead found that despite the high level of population divergence at neutral loci, the guppy populations studied here are more similar at the MHC than expected by neutral evolution (Fraser et al. 2009; also see Miller et al. 2001). Therefore, it is also possible that natural selection at the MHC and subsequent mate choice for MHC genotype can actually counteract speciation. Indeed, we have provided some of the first data implicating the widespread gyrodactylus pathogen as an agent of homogenizing selection across otherwise genetically disparate populations. Future work implicating adaptive mate choice for the MHC in the guppy could provide an important explanation for the apparent lack of reproductive barriers among the populations in Northern Trinidad.

Acknowledgments Su Youn Baek, Tim Hain, Rajendra Mahabir, Indar Ramnarine and Ho Young Suk assisted with field and laboratory work. Melissa Evans, Trevor Pitcher and two anonymous reviewers provided valuable comments on the manuscript. Funding was provided by Ontario Graduate Scholarship-Science and Technology to BAF and Natural Sciences and Engineering Research Council of Canada Discovery and Accelerator grants to BDN.

\section{References}

Cable J, van Oosterhout C (2007a) The role of innate and acquired resistance in two natural populations of guppies (Poecilia retiulata) infected with the ectoparasite Gyrodactylus turnbulli. Biol J Linn Soc 90:647-655

Cable J, van Oosterhout C (2007b) The impact of parasites on the life history evolution of guppies (Poecilia reticulata): the effects of host size on parasite virulence. Int J Parasitol 37:1449-1458

Carvalho GR, Shaw PW, Magurran AE, Seghers BH (1991) Marked genetic divergence revealed by allozymes among populations of the guppy Poecilia reticulata (Poeciliidae) in Trinidad. Biol J Linn Soc 42:389-405

Eizaguirre C, Lenz TL, Traulsen A, Milinksi M (2009) Speciation accelerated and stabilized by pleiotropic major histocompatibility complex immunogenes. Ecol Lett 12:5-12

Figueroa F, Mayer WE, Sultmann H, O'hUigin C, Tichy H, Satta Y, Takezaki N, Takahata N, Klein J (2000) MHC class II B gene evolution in east african cichlid fishes. Immunogenetics 51:557575

Fraser BA, Ramnarine IW, Neff BD (2009) Selection at the MHC class IIB locus across guppy (Poecilia reticulata) populations. Heredity. doi:10.1038/hdy2009.99

Houde AE (1997) Sexual selection and mate choice in guppies. Princeton University Press, Princeton, NJ

Kania P, Larsen TB, Ingerslev HC, Buchmann K (2007) Baltic salmon activates immune relevant genes in fin tissue when responding to Gyrodactylus salaris infection. Dis Aquat Org $76: 81-85$

Klein J (1990) The natural history of the major histocompatibility complex. Wiley, New York

Lindenstrøm T, Secombes CJ, Buchmann K (2004) Expression of immune response genes in rainbow trout skin induced by Gyrodactylus derjavini infections. Vet Immuol Immunopathol 97:137-148

Lyles AM (1990) Genetic variation and susceptibility to parasites: Poecilia reticulata infected with Gyrodactylus turnbulli. $\mathrm{PhD}$ thesis, Princeton University

Magurran AE (1998) Population differentiation without speciation. Phil Trans R Soc Lond B 353:275-286

Martin CH, Johnsen S (2007) A field test of the Hamilton-Zuk hypothesis in the Trinidadian guppy (Poecilia reticulata). Behav Ecol Sociobiol 61:1897-1909

McConnell TJ, Godwin UB, Norton SF, Nairn RS, Kazianis S, Morizot DC (1998) Identification and mapping of two divergent, unlinked major histocompatibillity complex class II $B$ genes in xiphophorus fishes. Genetics 149:1921-1934

Miller KM, Kaukinen KH, Beacham TD, Withler RE (2001) Geographic heterogeneity in natural selection on an MHC locus in sockeye salmon. Genetica 111:237-257

Ono H, O’hUigin C, Vincek V, Klein J (1993) Exon-intron organization of fish major histocompatibility complex class II $B$ genes. Immunogenetics 38:223-234

Ottová E, Šimková A, Morand S (2007) The role of major histocompatibility complex diversity in vigour of fish males (Abramis brama L.) and parasite selection. Biol J Linn Soc 90:525-538

Pitcher TE, Neff BD (2006) MHC class IIB alleles contribute to additive and nonadditive genetic effects on survival in Chinook salmon. Mol Ecol 15:2357-2365

Roberts RJ, Smail DA (2001) Laboratory methods. In: Roberts RJ (ed) Fish pathology, 3rd edn. W.B. Saunders, London, pp 380412

Schad J, Ganzhorn JU, Sommer S (2005) Parasite burden and constitution of major histocompatibility complex in the malagasy mouse lemus, Microcebus murinus. Evolution 59:439-450

Scott ME (1985) Dynamics of challenge infections of Gyrodactylus bullatarudis Turnbull (Monogenea) on guppies. J Fish Dis 8:495-503

Scott ME, Anderson RM (1984) The population dynamics of Gyrodactylus bullatarudis (Monogenea) within laboratory populations of the fish host Poecilia reticulata. Parasitology 89:159194

Suk HY, Neff BD (2009) Microsatellite genetic differentiation among populations of the Trinidadian guppy. Heredity 102:425-434

van Oosterhout (2009) A new theory of MHC evolution: beyond selection on the immune genes. Proc R Soc B 276:657-665 
van Oosterhout C, Joyce DA, Cummings SM, Blais J, Barson NJ, Ramnarine IW, Mohammed RS, Persad N, Cable J (2006) Balancing selection, random genetic drift, and genetic variation at the major histocompatibility complex in two wild populations of guppies (Poecilia reticulata). Evolution 60:2562-2574

van Oosterhout C, Mohammed RS, Hansen H, Archard GA, McMullan M, Weese DJ, Cable J (2007a) Selection by parasites in spate conditions in wild Trinidadian guppies (Poecilia reticulata). Int J Parasitol 37:805-812

van Oosterhout C, Smith AM, Hänfling B, Ramnarine IW, Mohammed RS, Cable J (2007b) The guppy as a conservation model: implications of parasitism and inbreeding for reintroduction success. Conserv Biol 21:1573-1583

van Oosterhout C, Potter R, Wright H, Cable J (2008) Gyro-scope: an individual-based computer model to forecast gyrodactylid infections on fish hosts. Int J Parasitol 38:541-548

Wegner KM, Kalbe M, Kurtz J, Reusch TBH, Milinski M (2003) Parasite selection for immunogenetic optimality. Science 301:1343

Fraser BA, Neff BD (in review) Additive and non-additive effects on fitness measures in the guppy 